\title{
Risk factors in Central Poland for the development of superficial and nodular basal cell carcinomas
}

Aleksandra Lesiak', Malgorzata Slowik-Rylska ${ }^{1}$, Michal Rogowski-Tylman ${ }^{1}$, Anna Sysa-Jedrzejowska' Mary Norval ${ }^{2}$, Joanna Narbutt ${ }^{1}$

1Department of Dermatology, Medical University of Lodz, Lodz, Poland 2Biomedical Sciences, University of Edinburgh Medical School, Edinburgh, Scotland

Submitted: 10 September 2008

Accepted: 22 October 2008

Arch Med Sci 2010; 6, 2: 270-275

DOI: 10.5114 /aoms.2010.13907

Copyright (c) 2010 Termedia \& Banach
Corresponding author:

Dr Aleksandra Lesiak Department of Dermatology Medical University of Lodz Krzemieniecka 5 94-017 Lodz, Poland Phone/fax: +48 426867981 +48426884565

E-mail: lesiak_ola@interia.pl

\begin{abstract}
Introduction: In the last decades the number of skin carcinomas has dramatically increased, which is mainly connected with changes in lifestyle, especially with common use of artificial light sources such as sunbeds. Basal cell carcinoma (BCC) is the most common form of skin cancer in white populations. Basal cell carcinomas are divided into subtypes, depending on their clinical picture and histology. The main groups are nodular (nBCC) and superficial (sBCC) ones. The major recognized risk factors for basal cell carcinoma (BCC) are exposure to chronic and intermittent burning doses of sunlight. Other risk factors leading to the development of the $\mathrm{nBCC}$ and $\mathrm{SBCC}$ subtypes of BCC are not well established. Material and methods: An analysis of 123 patients with either $\mathrm{nBCC}$ or SBCC, living in Lodz, Poland, regarding various intrinsic and environmental parameters was undertaken following the histological diagnosis of BCC.

Results: No statistical differences were observed between the BCC subtype and sex, age, hair colour, eye colour, smoking, family history of skin cancer, occupation, or past episodes of sunburn. While sBCCs tended to occur on unexposed body sites in phototype I/II subjects who mainly avoided direct sunlight, nBCCs tended to occur on sun-exposed body sites in phototype III subjects who were frequently in direct sunlight.

Conclusions: Thus the development of particular BCC subtypes is partially dependent on phototype and personal sun behaviour.
\end{abstract}

Key words: nodular basal cell carcinoma, skin phototype, sun exposure, superficial basal cell carcinoma.

\section{Introduction}

Basal cell carcinoma (BCC) is the most common form of skin cancer in white populations, with an average lifetime risk of developing BCC estimated at approximately $30 \%$ [1]. In general, the closer subjects live to the equator, the higher the incidence [2]. Sun exposure is a major risk factor for all BCCs [3], although the pattern of irradiation may be different for the various subtypes $[4,5]$. Intermittent intense sun exposure leading to sunburn episodes, especially during childhood and adolescence, is a consistent finding [6]. Cumulative sun exposure is likely to be an important additional risk factor [7]. Individuals with the highest risk of developing BCCs are usually fair-skinned and have a history of burning rather than tanning in response to solar ultraviolet radiation [8]. 
In several countries worldwide there has been a considerable increase in the incidence of BCCs in recent years. The highest increase in incidence rate is reported in Australia and is 1-2\% annually [9]. In the south-east Netherlands, an approximate doubling in BCC incidence from 2000 to 2015 is projected, due, in part, to ageing of the population [10]. In Poland there is a lack of statistical data concerning the prevalence of BCC but regional data from the north of the country indicate a recent increase in the incidence rate. The number of BCC cases is thought to be significantly under-reported as the lesions are often removed after clinical diagnosis but without histological analysis, and patient management is frequently undertaken by general practitioners in primary care rather than by dermatologists [11, 12]. In most European countries, BCCs are not included in national cancer registries so an accurate picture of BCC prevalence and any change in incidence over time is difficult to obtain.

The BCCs are divided into subtypes, depending on their histology, with the two main groups being nodular (nBCC) and superficial (sBCC) [13]. Nodular $\mathrm{BCC}$ is considered as non-aggressive while the other subtypes are associated with an increased risk of recurrence or local invasive behaviour. Other differences are reported. For example, patients with SBCC are generally younger than patients with $\mathrm{nBCC}$, the frequency of $\mathrm{BCC}$ is higher overall in men than in women but the frequency of SBCC is higher in women than in men, and BCCS on the head/neck are mainly nodular while those on the trunk are mainly superficial [13-15]. Because of these variables, BCC should not be considered as a single entity and identification of the risk factors involved in development of the subtypes may aid in the management of these lesions, which represent a significant and costly health burden as well as considerable cosmetic disfigurement.

The aim of the present study was to obtain information, by use of a questionnaire, about various personal and environmental risk factors in patients living in Lodz, central Poland, who had been diagnosed with either sBCC or nBCC.

\section{Material and methods}

The total number of patients biopsied and treated for a single BCC at the Department of Dermatology, Medical University of Lodz, between 2000 and 2006 was 445. A letter of invitation was sent to these individuals inviting them to participate in the study and 123 accepted. They were all Caucasian (53 M, $70 \mathrm{~F}$, mean age 68 years, range 49-86 years) and none was an organ transplant recipient, was being treated with immunosuppressive drugs or suffered from any other malignancy. Each subject gave written informed consent before entering the study which had been approved by the local Ethic Committee.

The individuals were given a questionnaire to complete which asked for details of sex, age, body site of BCC, hair colour ("fair" included red, blond and dark blond, and "dark" included brown and black), eye colour ("pale" included blue, grey and green, and "dark" included brown and black), smoking history, occupation, amount of time spent outside (hobbies, sport or work), frequency of sun exposure, use of sunbeds, number of lifetime sunburns, ability to tan in response to sunlight, and family history of skin cancer. In addition the subjects were assessed by a dermatologist for their skin phototype, according to the Fitzpatrick score [16]. They were assigned to phototype I/II (always sunburns, never or rarely tans, lack of or weak immediate and delayed pigmentation, and ivory white or white skin on unexposed body site), III (sometimes burns, always tans, visible immediate and delayed pigmentation and white skin on unexposed body site) or IV (never burns, always tans, moderate immediate or delayed pigmentation, moderate tan on unexposed body site). The results were analysed statistically by the Mann-Whitney and the $\chi^{2}$ tests, with $p<0.05$ considered as being significant.

\section{Results}

The results, based on the questionnaire, were analysed in relation to the histological diagnosis of sBCC or nBCC and are shown in Table I. The histology of the two types of lesions are illustrated in Figure 1. The superficial subtype of BCC was diagnosed in $22.7 \%$ of the 123 patients and $n B C C$ in $77.3 \%$. The mean diameter of the nBCCs was $0.9 \mathrm{~cm}$ (range $0.3-1.5 \mathrm{~cm}$ ) and of the sBCCs was $2.5 \mathrm{~cm}$ (range $0.5-7.0 \mathrm{~cm})(p<0.05)$. There was no statistical difference in the size of either of the two subtypes in men compared with women. There was no correlation between the histological subtype of BCC and the sex, age, hair colour, eye colour, smoking history, family history of skin cancer or outdoor activities of the patient $(p>0.05$ for all comparisons). In addition no significant difference was found between subtype and frequency of exposure to the sun during work, sport or hobbies $(p>0.05)$. The patients were asked whether they had been sunburnt at any time and, if so, on how many occasions. There was no correlation between the BCC subtype and the occurrence or lack of occurrence of sunburn during a lifetime $(p>0.05)$. It was found that the subjects who said that they had experienced sunburn could not recall on how many occasions during their lifetime this had happened. Thus they were divided into those who reported a single sunburn episode and those who reported more than one sunburn episode (Table I). 
Table I. Characteristics of patients diagnosed with either superficial or nodular basal cell carcinoma (BCC)

\begin{tabular}{|lll|}
\hline & Superficial BCC & Nodular BCC \\
\hline Number of subjects & 28 & 95 \\
\hline Mean age [years] & 69 & 65 \\
\hline Sex & F 18, M 10 & F 51, M 44 \\
\hline Hair colour & fair 18, dark 10 & fair 62, dark 33 \\
\hline Eye colour & pale 15, dark 13 & pale 56, dark 39 \\
\hline Smoking history & yes 14, no 14 14 , no 47 \\
\hline Family history of skin cancer & yes 17, no 11 & yes 48, no 13 \\
\hline Outdoor activities & yes 15, no 13 & yes 46, no 49 \\
\hline Exposure to the sun & frequent 13, rare 15 & yes 57, no 43 \\
\hline Occurrence of sunburn & yes 12: 5 on one occasion, & frequent 57, rare 38 \\
\hline Number with skin phototype & 7 on multiple occasions & 22 on multiple occasions \\
\hline Sunbed users & I/II 13, III 9, IV 6 & I/II 22, III 52, IV 21 \\
\hline Body site of BCC & yes 1 (phototype III), no 27 & yes 3 (phototype III), no 92 \\
\hline
\end{tabular}
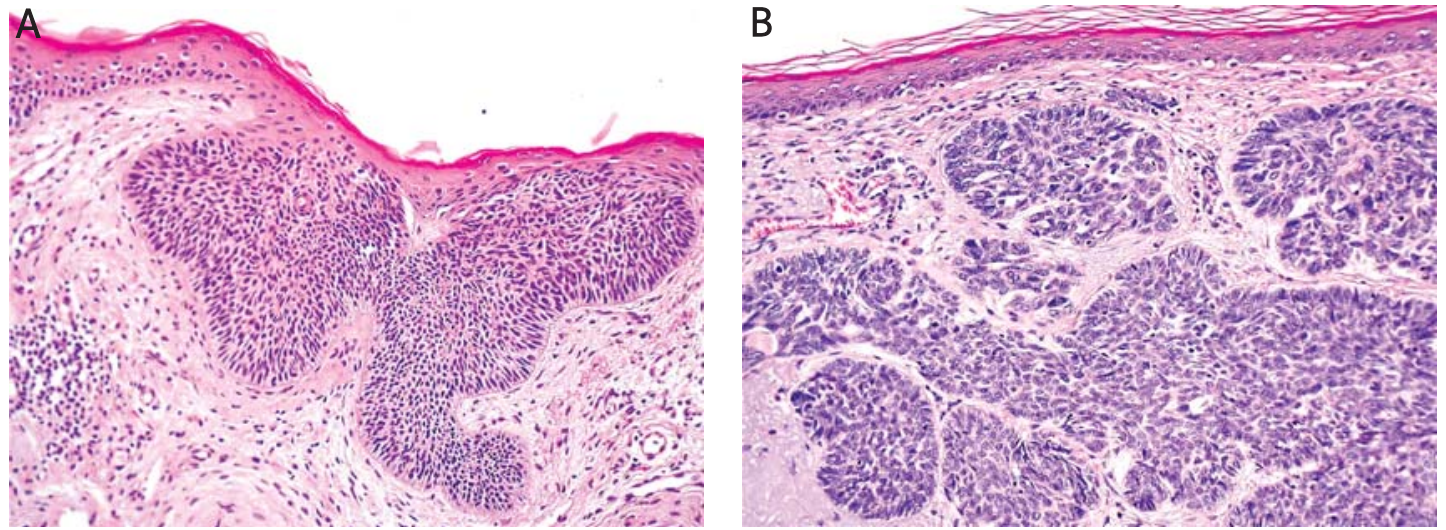

Figure 1. Histology of (A; mag. 200x) superficial (buds of basaloid cells attached to the undersurface of the epidermis, nests of various sizes in the upper dermis, tumour cell aggregates with typical peripheral palisading) and (B; mag. 200x) nodular (tumour cells with large, hyperchromatic, oval nuclei and sparse cytoplasm, various size of tumour nests, tumour cells align more densely in a palisade pattern at the periphery of these nests) subtypes of basal cell carcinoma

On analysis, no significant difference between the subtype of BCC and sunburn episodes was demonstrated $(p>0.05)$. When skin phototype was considered, it was revealed that SBCC occurred more frequently in those with phototype I/II than III or IV, and nBCC more frequently in those with phototype III than I/II or IV ( $p<0.05$ for both comparisons) (Figure 2). In $56 \%$ of patients the BCCs were located on sun-exposed body sites. Nodular BCCs were found more frequently (69.5\%) on sun-exposed areas (mainly the head and neck) than on unexposed areas $(p<0.005)$, while more sBCCs (89.3\%) were present on unexposed areas (the trunk) than on sun-exposed areas $(p<0.005)$ (Figure 3). There was no difference between males and females in the distribution of either nBCCs or sBCCs ( $p>0.05$ for both comparisons). The number of sunbed users was too small (Table I) to allow statistical analysis.

\section{Discussion}

During the seven-year period from 2000 to 2006, 445 new cases of BCC were diagnosed and treated in the Dermatology Department, Medical University of Lodz. Although this number represents an unknown proportion of the total number of BCCs in central Poland, a doubling in the annual incidence rate at our hospital was noted, from 46 cases in 2000 to 96 in 2006. This trend follows that noted in many other reports worldwide, such as in Britain [17], Brazil [18], Sweden [19], the Netherlands [10], Italy [20] and Australia [19]. Most studies indicate a higher risk for BCC development in the elderly, 


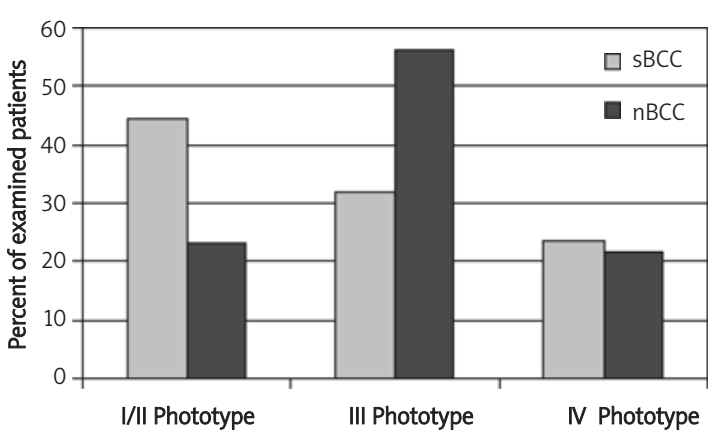

Figure 2. Phototype of the 123 patients who had either nodular (n) or superficial (s) subtype of basal cell carcinoma

although an increase in the number of cases in younger women has been noted recently [10, 17, 21]. This was not the case in our population, as the mean age of BCC cases in 2000 was 69.2 years (24 cases in women and 22 in men) and in 2006 was 68.8 years ( 50 cases in women and 46 in men). Basal cell carcinoma is reported to be more common in men than in women with a male : female ratio often quoted as around $2: 1[13,15,22$, 23]. In other studies the incidence in women is not significantly different from that in men [4, 25-28] and our findings fit with the latter results as only slightly more than half (56\%) of the total BCCS occurred in women.

When the subtypes of BCC were analysed histologically, $\mathrm{nBCC}$ was found in the majority (77.3\%) of cases. This result is consistent with the observations made by others [13, 24, 29], although in some surveys SBCCs outnumber nBCCs [reviewed in 25]. This discrepancy may be due to the method of collecting the BCC cases, as many SBCCs are treated outside a hospital setting and are not analysed histologically, thus leading to a smaller proportion than is actually present in the community. The size of the SBCCs was significantly larger than the size of the $\mathrm{nBCC}$ in our study. This may be explained by the sBCCs not being recognised clinically until a later stage than is the case for the nBCCs.

We were interested to try to relate age and sex to the occurrence of the subtypes of BCC, as an increasing incidence of sBCC in younger subjects, especially women, has been reported in the last decade [4, 24]. However, although we found that $64 \%$ of the cases of sBCC were in women, this was not a significant difference from $\mathrm{nBCC}$, perhaps due to the relatively small number of patients studied. In addition there was no age difference between those individuals developing SBCC (mean age of women 68.2 years, men 70.8 years) and $n B C C$ (mean age of women 66.1 years, men 64.9 years), unlike other reports where SBCC was diagnosed at a significantly lower age than in those with $\mathrm{nBCC}$

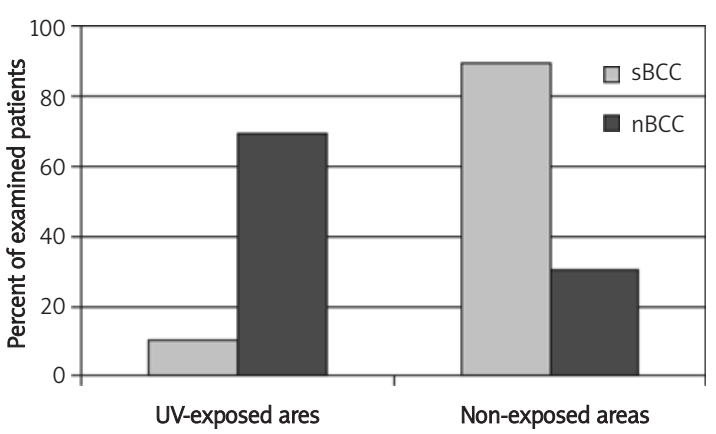

Figure 3. Body sites of the nodular (n) and superficial (s) basal cell carcinomas in 123 patients

$[4,13,24]$. Several groups have found that fair hair and pale eyes are risk factors for BCC $[14,30]$. In the present study we demonstrated that the majority of the patients with BCC had fair hair and pale eyes, but having these features did not correlate significantly with the development of sBCC or nBCC. Similarly, there was no correlation of BCC subtype with smoking history or a family history of BCC.

As cumulative and intermittent intense sun exposure are recognized as the most important environmental risk factors for BCC, the involvement of the sun in the development of BCC subtypes was examined in several ways, by asking first whether the patient's job was outdoors or indoors, secondly how much time the patients spent outside as a result of their hobbies or sport, and thirdly whether the patient remembered experiencing one or more sunburn episodes in the past. In no case was there a significant difference between these factors and the occurrence of sBCC or nBCC. However, individuals with SBCC were less likely to expose themselves frequently to the sun than those with $\mathrm{nBCC}$ and, when skin phototype was considered, sBCC occurred most often in subjects with phototype $\mathrm{I} / \mathrm{II}$ and $\mathrm{nBCC}$ in subjects with phototype III, indicating that the response of the individual to sun exposure may be a factor in determining the subtype of BCC that develops. Basal cell carcinomas have been reported to occur predominantly in those with phototype I/II, but the risk of $\mathrm{nBCC}$ or sBCC according to phototype has not been recorded previously. Basal cell carcinomas are found on body sites that are infrequently sunexposed as well as on chronically exposed skin, and the sites most commonly involved differ for the subtypes, with $\mathrm{nBCC}$ developing mainly on the head and neck and sBCC mainly on the trunk [4, 27, 29, 31]. This was confirmed in the present study, with almost $70 \%$ of $n B C C$ s on sun-exposed areas (head and neck) and $89 \%$ of sBCCs on less sun-exposed areas (trunk). We found that the majority of the nBCC patients tended to expose themselves frequently to the sun, while the majority of the 
sBCC patients tended to avoid sun exposure. This behavioural difference may be explained to some extent by phototype, as $46 \%$ of the sBCC patients, but only $23 \%$ of the nBCC patients, were phototype I/II. In contrast, Neale et al. [15] reported a positive association between sun sensitivity and BCC of the head (predominantly $n B C C$ ), but this was absent for BCC of the trunk (predominantly sBCC). Their study was based in Nambour, Queensland, a location with much higher solar ultraviolet radiation than central Poland, and perhaps the difference in UV intensity and cumulative dose may be important variables in determining the subtype of BCC that develops and its body site. No significant difference in the site distribution of the BCC subtypes between men and women was demonstrated in our study group, a result also reported by Bernard et al. [25] but not by Bastiaens et al. [4], who found a slightly higher number of sBCCs on the trunk in males (55\%) compared with females in a review that contained a larger number of patients with SBCC (302) than ours. While the use of sunbeds has been suggested as contributing to BCC induction (32), this was not an important factor in the present study, as only $3 \%$ of the individuals with BCC had used such tanning devices. Sunbeds are not popular in Poland and were not widely available in the last decades of the twentieth century.

In conclusion, our results reveal that, in central Poland, nBCCs tend to occur on exposed body sites in many subjects with phototype III who are frequently in direct sunlight, while SBCCs tend to occur on unexposed body sites in many subjects with phototype I/II who frequently avoid direct sunlight. Thus the development of the BCC subtype may be partially dependent on phototype and personal sun behaviour.

\section{Acknowledgments}

The study was funded by an internal grant from the Medical University of Lodz, no. 503-1019-1 and project number NN403474737. The authors declare no conflict of interest. The study was done in Poland.

\section{References}

1. Lear JT, Schmith AG. Basal cell carcinoma. Postgrad Med J 1997; 73: 538-42

2. Swanbeck G, Hillstrom L. Analysis of etiological factors of squamous cell skin cancer of different locations. Acta Derm Venereol 1971; 51: 151-6.

3. Leiter U, Garbe C. Epidemiology of melanoma and nonmelanoma skin cancer - the role of sunlight. Adv Exp Med Biol 2008; 624: 89-103.

4. Bastiaens MT, Hoefnagel JJ, Bruijn JA, Westendorp RG, Vermeer BJ, Bouwes-Bavinck JN. Differences in age, site distribution, and sex between nodular and superficial basal cell carcinomas indicate different types of tumors. J Invest Dermatol 1998; 110: 880-4.
5. Gruber F, Zamolo G, Kastelan M, et al. Photocarcinogenesis - molecular mechanisms. Coll Antropol 2007; 31 (suppl 1): 101-6.

6. Kricker A, Armstrong BK, English DR, Heenan PJ. Does intermittent sun exposure cause basal cell carcinoma? A case-control study in Western Australia. Int J Cancer 1995; 60: 489-94.

7. Leman JA, McHenry PM. Basal cell carcinoma: still an enigma. Arch Dermatol 2001; 137: 1239-40.

8. Green A, Battistutta D, Hart V, Leslie D, Weedon D. Skin cancer in a subtropical Australian population: incidence and lack of association with occupation. The Nambour Study Group. Am J Epidemiol 1996; 144: 1034-40.

9. Roewert-Huber J, Lange-Asschenfeldt B, Stockfleth E, Kerl H. Epidemiology and aetiology of basal cell carcinoma. Br J Dermatol 2007; 157 (Suppl 2): 47-51.

10. de Vries E, Louwman M, Bastiaens M, de Gruijl FR, Coebergh JW. Rapid and continuous increases in incidence rates of basal cell carcinoma in the southeast Netherlands since 1973. J Invest Dermatol 2004; 123: 634-8.

11. Diepgen TL, Mahler V. The epidemiology of skin cancer. $\mathrm{Br}$ J Dermatol 2002; 146 (Suppl 61): 1-6.

12. Rubin Al, Chen EH, Ratner D. Basal cell carcinoma. N Engl J Med 2005; 353: 2262-9.

13. Raasch BA, Buettner PG, Garbe C. Basal cell carcinoma: histological classification and body-site distribution. $\mathrm{Br}$ J Dermatol 2006; 155: 401-7.

14. Zanetti R, Rosso S, Martinez C, et al. Comparison of risk patterns in carcinoma and melanoma of the skin in men: a multi-centre case-case-control study. Br J Cancer 2006; 94: 743-51.

15. Neale RE, Davis M, Pandeya N, Whiteman DC, Green AC. Basal cell carcinoma on the trunk is associated with excessive sun exposure. J Am Acad Dermatol 2007; 56: 380-6.

16. Fitzpatrick TB. The validity and practicality of sun-reactive skin types I through VI. Arch Dermatol 1988; 124: 869-71.

17. Bath-Hextall F, Leonardi-Bee J, Smith C, Meal A, Hubbard R. Trends in incidence of skin basal cell carcinoma. Additional evidence from a UK primary care database study. Int J Cancer 2007; 121: 2105-8.

18. Nasser N. Epidemiology of basal cell carcinomas in Blumenau, SC, Brazil, from 1980 to 1999. An Bras Dermatol 2005; 80: 363-8.

19. Wassberg C, Thörn M, Johansson AM, Bergström R, Berne $B$, Ringborg $U$. Increasing incidence rates of squamous cell carcinoma of the skin in Sweden. Acta Derm Venereol 2001; 81: 268-72.

20. Delfino S, Innocenzi D, Di Lorenzo G, et al. An increase in basal cell carcinoma among the young: an epidemiological study in a middle-south Italian population. Anticancer Res 2006; 26: 4979-83.

21. Staples MP, Elwood M, Burton RC, Williams JL, Marks R, Giles GG. Non-melanoma skin cancer in Australia: the 2002 national survey and trends since 1985. Med J Aust 2006; 184: 6-10.

22. Ceylan C, Oztürk G, Alper S. Non-melanoma skin cancers between the years of 1990 and 1999 in Izmir, Turkey: demographic and clinicopathological characteristics. J Dermatol 2003; 30: 123-31.

23. Coups EJ, Manne SL, Heckman CJ. Multiple skin cancer risk behaviors in the U.S. population. Am J Prev Med 2008; 34: 87-93.

24. McCormack CJ, Kelly JW, Dorevitch AP. Differences in age and body site distribution of the histological subtypes of basal cell carcinoma. A possible indicator of differing causes. Arch Dermatol 1997; 133: 593-6. 
25. Bernard P, Dupuy A, Sasco A, et al. Basal cell carcinomas and actinic keratoses seen in dermatological practice in France: a cross-sectional survey. Dermatology 2008; 216: 194-9.

26. Czarnecki D, Collins N, Meehan C, O'Brien T, Leahy S, Nash C. Basal-cell carcinoma in temperate and tropical Australia. Int J Cancer 1992; 50: 874-5.

27. Betti R, Inselvini E, Carducci M, Crosti C. Age and site prevalence of histologic subtypes of basal cell carcinomas. Int I Dermatol 1995; 34: 174-6.

28. Cho S, Kim MH, Whang KK, Hahm JH. Clinical and histopathological characteristics of basal cell carcinoma in Korean patients. J Dermatol 1999; 26: 494-501.

29. Scrivener Y, Grosshans E, Cribier B. Variations of basal cell carcinomas according to gender, age, location and histopathological subtype. Br J Dermatol 2002; 147: 41-7.

30. Lear JT, Tan BB, Smith AG, et al. Risk factors for basal cell carcinoma in the UK: case-control study in 806 patients. J R Soc Med 1997; 90: 371-4.

31. Lovatt TJ, Lear JT, Bastrilles J, et al. Associations between ultraviolet radiation, basal cell carcinoma site and histology, host characteristics, and rate of development of further tumors. J Am Acad Dermatol 2005; 52: 468-73.

32. Faurschou A, Wulf HC. Ecological analysis of relation between sunbeds and skin cancer. Photodermatol Photoimmunol Photomed 2007; 4: 120-5. 